\title{
Análise do ambiente educacional de uma escola médica brasileira à luz das demandas
}

\section{do ensino remoto}

Analysis of the educational environment of a brazilian medical school in light of the demands of remote learning

Análisis del entorno educativo de una escuela de medicina brasileña a la luz de las demandas de la enseñanza a distancia

Sandra Torres Serra

ORCID: https://orcid.org/0000-0001-8303-5732 Universidade do Estado do Rio de Janeiro, Brasil

E-mail: sandratserra@gmail.com

Stella Regina Taquette ORCID: https://orcid.org/0000-0001-7388-3025 Universidade do Estado do Rio de Janeiro, Brasil E-mail: stella.taquette@gmail.com

Nádia Cristina Pinheiro Rodrigues ORCID: https://orcid.org/0000-0002-2613-5283 Universidade do Estado do Rio de Janeiro, Brasil E-mail: nadiacristinapr@gmail.com

Letícia Moraes Corrêa ORCID: https://orcid.org/0000-0003-2116-4613 Universidade do Estado do Rio de Janeiro, Brasil E-mail: letmorales.correa@gmail.com

Mariana Bteshe

ORCID: https://orcid.org/0000-0002-3456-079X Universidade do Estado do Rio de Janeiro, Brasil E-mail: marianabteshe@gmail.com

\begin{abstract}
Resumo
A necessidade de distanciamento social provocou impactos na formação médica, que já sofria processo de mudanças. Com a adoção do ensino remoto, ocasionada pela pandemia da Covid-19, as escolas médicas revisaram práticas educativas e a relação professor-aluno em novos cenários. Nosso objetivo é analisar o ambiente educacional de um curso de graduação de medicina contextualizando-o à nova realidade, identificando aspectos do curso que devem ser revistos para favorecer as transformações necessárias. Aplicamos questionários sociodemográficos e Dundee Ready Education Environment Measure (DREEM) a alunos do primeiro, terceiro e sexto anos do curso de medicina de uma universidade pública brasileira entre agosto e novembro de 2019. Os alunos avaliaram negativamente o ambiente educacional, sendo aspectos concernentes à motivação, uso inadequado do tempo, incentivo à educação permanente percebidos como pouco estimulados e que precisam ser melhorados, objetivos pedagógicos pouco esclarecidos e professores autoritários. Na análise dos dados destacamos as percepções dos alunos sobre motivação e estímulo para aprender; relação professor-aluno; autonomia e estímulo à educação continuada; avaliação, e prática de feedback. Os resultados indicam que a adoção de metodologias de ensino centradas no professor não favorece a participação ativa do aluno e não o encoraja à construção do conhecimento. A modalidade remota de ensino exige ressignificar práticas educativas e lugares ocupados por professores e alunos no processo de ensino-aprendizagem. Atividades de aprimoramento docente, revisão dos métodos de ensino e de avaliação, e maior participação de alunos e professores nos processos de mudança são necessárias.
\end{abstract}

Palavras-chave: COVID-19; Educação de Graduação em Medicina; Ensino Online; Avaliação curricular das escolas médicas; Estudantes.

\section{Abstract}

The need for social distance caused impacts on medical education, which was already undergoing a process of changes. The use of remote teaching, caused by the Covid-19 pandemic, required medical schools to review educational practices and the teacher-student relationship in new scenarios. Our aim is to analyze the educational environment of an 
undergraduate medical course contextualizing it to the new reality, identifying aspects of the course that should be reviewed to foster the necessary transformations. Sociodemographic questionnaires and Dundee Ready Education Environment Measure (DREEM) were applied to students from the first, third and sixth years of the medical undergraduate course of a Brazilian public university from August to November 2019. The educational environment was negatively evaluated by the students, with aspects concerning motivation, inadequate use of time, encouragement of continuing education perceived as little stimulated and in need of improvement, unclear pedagogical goals and authoritarian teachers. Students' perceptions of motivation and encouragement to learn; teacher-student relationship; autonomy and encouragement to continuing education; evaluation, and feedback practice were highlighted in the data analysis. The adoption of teaching methodologies centered on the teacher does not favor the active participation of the student and does not encourage them to build knowledge. The remote modality of teaching requires a redefinition of educational practices and the places occupied by teachers and students in the teaching-learning process. Teacher improvement activities, review of teaching and assessment methods, and greater participation of students and teachers in change processes are necessary.

Keywords: COVID-19; Medical education undergraduate; Education online; Evaluation of medical school curriculum; Students.

\section{Resumen}

La necesidad de distanciamiento social incidió en la formación médica, que ya estaba en un proceso de cambio. Con la adopción del aprendizaje remoto, provocada por la pandemia Covid-19, las escuelas de medicina revisaron las prácticas educativas y la relación profesor-alumno en nuevos escenarios. Nuestro objetivo es analizar el entorno educativo de un curso de graduación de medicina contextualizándolo a la nueva realidad, identificando aspectos del curso que deben ser revisados para favorecer los cambios necesarios. Se aplicaron cuestionarios sociodemográficos y Dundee Ready Education Environment Measure (DREEM) a estudiantes de medicina de primero, tercero y sexto año de una universidad pública brasileña entre agosto y noviembre de 2019. Los estudiantes evaluaron negativamente el entorno educativo, con aspectos relacionados con la motivación, inadecuado aprovechamiento del tiempo, fomento de la educación permanente percibida como poco estimulada y con necesidad de mejora, objetivos pedagógicos poco aclarados y docentes autoritarios. En el análisis de datos, destacamos las percepciones de los estudiantes sobre la motivación y el estímulo para aprender; relación profesor-alumno; autonomía y estímulo a la educación continua; práctica de evaluación y retroalimentación. Los resultados indican que la adopción de metodologías de enseñanza centradas en el docente no favorece la participación activa del alumno y no incentiva la construcción del conocimiento. La modalidad de enseñanza a distancia requiere una redefinición de las prácticas educativas y los lugares que ocupan los docentes y estudiantes en el proceso de enseñanza-aprendizaje. Son necesarias actividades de mejora docente, revisión de los métodos de enseñanza y evaluación, y una mayor participación de estudiantes y docentes en los procesos de cambio.

Palabras clave: COVID-19; Educación de pregrado en medicina; Educación en línea; Evaluación de los planes de estudios de las escuelas de medicina; Estudiantes.

\section{Introdução}

\section{Necessidade de mudanças no contexto da Educação Médica atualizada pela pandemia}

O modelo flexneriano de ensino (fragmentação dos currículos, ênfase conteudista e papel passivo dos estudantes como meros reprodutores do conhecimento) vem sendo questionado nas últimas décadas (Behrens, 1999; Rodríguez, Poli \& Behrens, 2004), e documentos ligados a organizações nacionais e internacionais recomendam mudanças na educação dos profissionais de saúde em todo o mundo (Ministério da Educação do Brasil., 2014); Frenk et al., 2010) Há um consenso de que tendências pedagógicas inovadoras deveriam nortear o campo da educação médica (Venturelli, 1997) de forma a melhor preparar os profissionais para os inúmeros e incertos desafios que se apresentam aos cuidados de saúde para este século (Frenk et al., 2010). No Brasil, essa tendência inovadora se traduz nas Diretrizes Curriculares para o curso de Medicina, que preconiza uma formação acadêmica cuja estrutura curricular seja centrada no estudante, considere as necessidades de saúde das comunidades e dos contextos em que os cursos se inserem, seja pautada na integração dos ciclos básico e clínico e de conteúdos teóricos com prática; na incorporação de metodologias ativas e problematizadoras de aprendizagem, em vivências continuadas em cenários de prática diversificados (Ministério da Educação do Brasil., 2014). Aprendizagem significativa e pensamento crítico que favoreçam a 
autonomia progressiva do aluno e o incentive à educação continuada e ao trabalho em equipe estariam contemplados nos métodos inovadores e tidos como indispensáveis para a atuação profissional futura.

Na eclosão da pandemia da Covid-19, as reformas que estavam em curso na formação dos profissionais de saúde (Frenk et al., 2010) foram redimensionadas de forma mais intensa e em escala global (Gordon et al., 2020) impulsionadas pela necessidade de incorporação de tecnologias de informação e comunicação (TICs) às práticas educativas. Além de desafios técnicos, operacionais e sociais para o uso de plataformas digitais (Alves, 2021), a mudança repentina para o ensino neste formato reposicionou a comunidade acadêmica frente às questões pedagógicas que já eram objeto de debates na formação em saúde antes da crise sanitária, como a motivação no processo de ensino-aprendizagem (Frenk et al., 2010; Genn \& Harden, 1986; Hutchinson, 2003)', metodologias para o aprendizado significativo (Behrens, 1999; Frenk et al., 2010; Ministério da Educação do Brasil.2014), papel do professor na contemporaneidade (Freire, 2005; Frenk et al., 2010; Ministério da Educação do Brasil.2014), e valor das interações sociais na formação acadêmica em saúde (Frenk et al., 2010).

Atualmente estima-se um progressivo retorno às aulas presenciais com possibilidades de incorporação do ensino remoto para algumas atividades (Dost, Hossain, Shehab, Abdelwahed \& Al-Nusair, 2020; Ruiz, Mintzer \& Leipzig, 2006), de forma que o ensino em modalidade híbrida (que conjuga momentos presenciais e remotos) apresenta-se como uma provável proposta pedagógica.

O uso ampliado de tecnologia online parece conduzir a uma reconfiguração da educação médica, inclusive no treinamento clínico (Dost et al., 2020; Emanuel, 2020) como verificado em experiências recentes (Gordon et al., 2020, Magalhães et al., 2020).

O ensino na modalidade remota, contudo, não se faz através da mera transposição de métodos tradicionais para o ambiente virtual (Goudouris \& Struchiner, 2015) pois implica em certas condições do ambiente educativo alinhadas a modelos mais inovadores, como relações professor-aluno mais horizontalizadas, uso de metodologias problematizadoras, prática regular de feedback e novas formas de avaliação do aprendizado (Venturelli, 1997; Organização das Nações Unidas para Educação, Ciência e Cultura [UNESCO], 2013). O docente precisa ter uma visão abrangente de plataformas e tecnologias online para fazer adequações ao ambiente virtual (O'Doherty et al., 2018;Organização das Nações Unidas para Educação, Ciência e Cultura [UNESCO], 2013); assim como o discente precisa desenvolver habilidades para se comunicar adequadamente com seus pares e trabalhar de forma colaborativa. Competências técnicas e relacionais, do docente e do discente, são fundamentais para que o ensino se estabeleça adequadamente na modalidade remota, portanto.

Diante destas constatações, nos questionamos se o cenário educativo da escola médica em estudo seria favorável às mudanças e se poderíamos dimensionar os desafios para responder às demandas de inovação. O objetivo deste trabalho foi analisar seu ambiente educacional em um momento prévio à eclosão da pandemia, à luz das novas questões provocadas pelo isolamento social e incorporação do ensino remoto, destacando os aspectos que deveriam ser revistos para a realização de transformações.

O ambiente educacional é um construto multifatorial que contempla a percepção do ensino, dos professores, dos vínculos interpessoais e da atmosfera organizacional (McAleer \& Roff, 2001; Troncon, 2014). É comprovada a importância da compreensão do ambiente para o desenvolvimento da aprendizagem no manejo das mudanças nas profissões de saúde (Genn \& Harden, 1986; Roff, 2005). Os dados gerados podem contribuir para os processos de avaliação mais amplos do curso, subsidiando decisões que se referem à formulação de intervenções ou inovações, aumentando a qualidade dos processos educativos (Genn, 2001; Troncon, 2014). 


\section{Metodologia}

Em 2019, empreendemos um estudo de natureza quanti-qualitativa com objetivo de investigar a percepção de docentes e discentes sobre o ambiente educacional de um curso de graduação em medicina de uma faculdade pública do Estado do Rio de Janeiro. Apesar de se encontrar em processo de mudança, o curso ainda dispõe de estrutura curricular tradicional, baseada no paradigma flexineriano.

Realizamos grupos focais com alunos e entrevistas com professores a fim de detectar os pontos fortes e as fragilidades da instituição e identificar aspectos que deveriam ser reformulados ou consolidados, visando aprimorá-la e adequá-la conforme às Diretrizes Curriculares Nacionais (DCN) de 2014.

Além de coletarmos dados através de entrevistas em grupos focais no período de agosto a novembro de 2019 , aplicamos, a uma amostra de alunos do primeiro, terceiro e sexto anos, o questionário Dundee Ready Education Environment Measure (DREEM) (Roff et al., 1997), instrumento que permite avaliar as percepções dos estudantes sobre o ambiente educacional em cinco dimensões: percepções do ensino, dos professores, dos resultados acadêmicos, da atmosfera e das relações sociais. Junto ao DREEM, aplicamos aos mesmos alunos um questionário sociodemográfico. Neste manuscrito, apresentamos os resultados quantitativos desta investigação e os analisamos compreensivamente à luz das transformações provocadas pelo isolamento social da pandemia de COVID19. Trata-se de um estudo descritivo e analítico, do tipo corte transversal (Gil, 2016; Taquette e Borges, 2010).

A participação dos alunos foi facultativa e todos assinaram o Termo de Consentimento Livre e Esclarecido (TCLE). No Brasil, esta pesquisa foi aprovada pelo Comitê de Ëtica do Hospital Universitário Pedro Ernesto/Universidade do Estado do Rio de Janeiro (protocolo - CAEE n ${ }^{\circ} 53535915.2 .0000 .5259$, parecer $n^{\circ} 1.542 .111$ ).

\subsection{Coleta de dados}

Os pesquisadores aplicaram os questionários de forma presencial, em um único momento para cada participante, no início das aulas de Anatomia (primeiro ano), Farmacologia (terceiro ano) e Clínica Médica (sexto ano). Orientamos os alunos quanto aos objetivos da pesquisa, preenchimento dos instrumentos e tempo máximo de 30 minutos para respondê-los de forma anônima e individual.

\subsection{Instrumentos}

1) Questionário sociodemográfico, composto por 16 perguntas fechadas e 2) Questionário DREEM, composto por 50 afirmações categorizadas em cinco subescalas. Cada uma das afirmações (itens) é pontuada em uma escala tipo Likert de cinco pontos com escores que variam de zero ("discordo fortemente") a quatro ("concordo fortemente"). A codificação reversa é necessária para alguns itens e as pontuações mais altas indicam uma percepção mais positiva do ambiente educacional. $\mathrm{O}$ DREEM tem pontuação máxima de 200 (ambiente educacional ideal) e quanto mais próximo desse valor estiver o resultado, mais positiva é a percepção (McAleer \& Roff, 2001).

\subsection{Análise dos dados}

Para a análise, seguimos o manual de utilização DREEM (McAleer \& Roff, 2001). Calculamos as pontuações totais e as médias obtidas para os itens e dimensões (subescalas). A pontuação média obtida para cada item revelou os pontos fortes e pontos fracos do ambiente educacional. Cada item possui o escore máximo de 4 pontos. Os itens com pontuação média menor que 2 são considerados problemáticos e necessitam de intervenção; maior que 3 corresponde a pontos fortes; e maior que 3,5 são 
áreas muito fortes. A pontuação entre 2 e 3 corresponde a aspectos do ambiente que podem ser melhorados pela instituição. Cada dimensão tem uma pontuação máxima e uma classificação de acordo com a pontuação alcançada. Para investigar a confiabilidade do instrumento, acessando sua consistência interna, calculamos o Alpha de Cronbach para todas as turmas. Além das médias, calculamos os desvios padrões da pontuação do DREEM. Utilizamos testes estatísticos para comparar as médias. O nível de significância considerado nos testes de diferenças de média foi de 0.05 . Utilizamos modelos binomiais negativos simples e ajustados para investigar a relação da turma e das características sócio demográficas com os resultados globais do DREEM (desfecho). As variáveis explicativas para o modelo ajustado foram: "Turma” (1ªno; $3^{\circ}$ ano; e $6^{\circ}$ ano), "Gênero" (Masculino; Feminino), "Idade" (<21; 21-23; 23-26; >; = 26), "Morava no Rio" (Sim; Não), "Mora com os pais" (Sim; Não), "Tempo CasaFaculdade" (até $30 \mathrm{~min}$; mais que $30 \mathrm{~min}$ ) "Renda Familiar” (<4.151,00; 4.151,00 - 8.300,00; 8.301,00 - 12.450,00; > 12.450,00), “Trabalha” (Sim; Não), "Ingresso na universidade por cotas de inclusão social” (Sim; Não) e "Número de vezes que prestou vestibular" (1 vez; 2 vezes; 3 vezes; 4 vezes ou mais). A seleção das variáveis do modelo ajustado levou em consideração o nível de significância da regressão simples (p-valores maiores que 0,20) e a coerência com a literatura científica.

As análises estatísticas foram realizadas através do software estatístico software R-Project versão 4.0.2.

Destacamos, na análise dos dados, os aspectos do ambiente educacional que consideramos fundamentais face aos novos desafios na educação médica advindos com o ensino remoto e as medidas de distanciamento social: percepções dos alunos sobre questões como motivação e estímulo para aprender; relação professor-aluno; autonomia e estímulo à educação continuada; avaliação; e prática de feedback, nos pareceram fundamentais a serem observados para avaliarmos sobre qual cenário as demandas de mudanças incidiram.

\section{Resultados}

Aplicamos 182 questionários a alunos do primeiro, terceiro e sexto anos do curso de graduação de medicina. Destes, descartamos 39 por erros de preenchimento (rasuras, duplicidade de respostas ou respostas em branco). No total, incluímos 143 questionários corretamente preenchidos $\left(1^{\circ}\right.$ ano $n=50 ; 3^{\circ}$ ano $n=70,6^{\circ}$ ano $\left.n=23\right)$, sendo 61 questionários respondidos por sujeitos do sexo masculino e 79, do sexo feminino. No questionário sociodemográfico, removemos da análise dados sem preenchimento. Dois sujeitos não preencheram adequadamente a informação sobre gênero e não puderam ser identificados, e um sujeito respondeu como "transgênero", e optamos por não incluí-lo nas análises que possuem a variável sexo.

Considerando que o mesmo ambiente educacional pode ser percebido de modos diferentes pelos estudantes de acordo com algumas variáveis (McAleer \& Roff, 2001; Roff \& McAleer, 2001), os dados sociodemográficos foram relacionados aos resultados do questionário DREEM. O Alpha de Cronbach encontrado foi 0,86 (Intervalo de Confiança de 95\%: 0,82 - 0,89), que é considerado satisfatório. Avaliamos os resultados do DREEM separadamente por ano e os analisamos comparativamente em três níveis: pontuação total, subescalas e itens individuais.

\subsection{Resultados Pontuação Total e Questionário Sociodemográfico}

O guia de interpretação do DREEM (McAleer \& Roff, 2001) classificou o ambiente educativo da instituição em estudo com “muitos problemas" (plenty of problems), com pontuação global de 95,03/200 pontos. Alunos do $1^{\circ}$ ano tiveram pontuação média maior em relação aos outros anos analisados (103,36 vs. 91,88 e 90,26 pontos - Tabela 1), com pouca diferença na pontuação entre alunos do $3^{\circ}$ e $6^{\circ}$ anos. Não houve diferença significativa entre a média da pontuação do DREEM e as características: gênero, faixa etária, raça, renda familiar, trabalhar, e ingressar na universidade por meio de cotas de inclusão 
social. Alunos que não moram com os pais, que antes de ingressar na faculdade moravam fora do estado, e que levam mais tempo para chegar à faculdade avaliaram melhor o curso de medicina.

Tabela 1 - Distribuição da pontuação total do DREEM por ano e segundo características sociodemográficas.

\begin{tabular}{|c|c|c|c|}
\hline \multirow[t]{2}{*}{ Variáveis } & \multicolumn{3}{|c|}{ Pontuação Total DREEM } \\
\hline & $\mathrm{N}$ & Média (desvio padrão) & P-valor \\
\hline Turma & & & 0,01 \\
\hline $1^{\circ}$ ano & 50 & $103,36(22,15)$ & \\
\hline $3^{\circ}$ ano & 70 & $91,88(20,55)$ & \\
\hline $6^{\circ}$ ano & 23 & $90,26(23,46)$ & \\
\hline Gênero & & & 0,16 \\
\hline Masculino & 61 & $98,93(25,19)$ & \\
\hline Feminino & 79 & $93,39(19,71)$ & \\
\hline Idade & & & 0,83 \\
\hline$<21$ & 51 & $96,98(21,80)$ & \\
\hline $21-23$ & 30 & $93,03(20,55)$ & \\
\hline $23-26$ & 34 & $94,44(21,51)$ & \\
\hline$>=26$ & 28 & $97,43(25,98)$ & \\
\hline Morava no Rio antes ${ }^{\mathrm{a}}$ & & & 0,01 \\
\hline Sim & 64 & $90,51(22,56)$ & \\
\hline Não & 55 & $101,42(21,56)$ & \\
\hline Mora com os pais & & & 0,003 \\
\hline Sim & 91 & $91,34(21,16)$ & \\
\hline Não & 51 & $102,78(22,21)$ & \\
\hline Tempo casa/faculdade & & & 0,04 \\
\hline$<=30 \mathrm{~min}$ & 60 & $99,93(21,30)$ & \\
\hline$>30 \mathrm{~min}$ & 82 & $92,17(22,32)$ & \\
\hline Renda familiar ${ }^{\mathrm{b}}$ & & & 0,59 \\
\hline
\end{tabular}




$\begin{array}{lcc}\text { Baixa } & 71 & 97,35(22,75) \\ \text { Média } & 25 & 93,8(22,84) \\ \text { Média-alta } & 22 & 90,23(21,02) \\ \text { Alta } & 24 & 95,62(19,89)\end{array}$

Trabalha

Sim

Não

$\begin{array}{lll}\text { Sim } & 65 & 98,66(21,98) \\ \text { Não } & 77 & 92,79(22,15)\end{array}$

Nota. $\mathrm{N}$ = Número de estudantes; DREEM = Escala Dundee Ready Education Environment Measure ${ }^{a}$ Morava no Estado do Rio de Janeiro antes de ingressar na UERJ;

'Renda Familiar: Baixa: < 4.151,00; Média: 4.151,00-8.300,00; Média Alta: 8.301,00-12.450,00; Alta: $>12.450,00$

Fonte: Produzida pelos autores.

\subsection{Resultados Subescalas - Dimensões}

Os resultados das médias das dimensões revelaram uma percepção negativa do ensino para os alunos do primeiro, terceiro e sexto anos, tal como ministrado na instituição em 2019. Alunos do primeiro ano têm percepção mais positiva do ambiente geral (atmosfera) do que os do terceiro e sexto anos. Nos três grupos, somente os alunos do primeiro ano se sentem confiantes quanto a seus resultados acadêmicos. Em relação à percepção dos professores, em média, foi avaliada a necessidade de aprimoramento. Na percepção das relações sociais, a faculdade foi considerada como um lugar "não agradável” (Quadro 1).

Quadro 1 - Distribuição da média de pontos das dimensões e percentual de pontos em cada ano.

\begin{tabular}{|cccc|}
\hline \multicolumn{4}{|c|}{ PERCEPÇÃO ENSINO (APRENDIZAGEM) - 48 pts } \\
\hline Média (IC 95\%) & Interpretação & Percentual $($ IC 95\%) \\
\hline $\mathbf{1}^{\mathbf{0}}$ ano & $23,04(21,30-24,76)$ & O ensino é visto de forma negativa & $48 \%(44 \%-52 \%)$ \\
\hline $\mathbf{3}^{\mathbf{0}}$ ano & $18,19(16,65-19,72)$ & O ensino é visto de forma negativa & $37 \%(35 \%-41 \%)$ \\
\hline $\mathbf{6}^{\mathbf{0}}$ ano & $20,30(17,08-23,53)$ & O ensino é visto de forma negativa & $42 \%(36 \%-49 \%)$ \\
\hline MÉDIA & $20,22(19,10-21,35)$ & O ensino é visto de forma negativa & $42 \%(40 \%-44 \%)$ \\
\hline
\end{tabular}




\begin{tabular}{|c|c|c|c|}
\hline \multicolumn{4}{|c|}{ PERCEPÇÃO DOS PROFESSORES - 44 pts } \\
\hline Ano & Média (IC 95\%) & Interpretação & Percentual $^{\mathrm{a}}(\mathrm{IC}$ 95\%) \\
\hline $1^{\circ}$ ano & $23,98(22,29-25,67)$ & Movendo-se na direção certa & $55 \%(51 \%-58 \%)$ \\
\hline $3^{\circ}$ ano & $23,86(22,65-25,07)$ & Movendo-se na direção certa & $54 \%(51 \%-57 \%)$ \\
\hline $6^{\circ}$ ano & $20,26(17,66-22,87)$ & Precisam de algum retreinamento & $46 \%(40 \%-52 \%)$ \\
\hline MÉDIA & $23,32(22,39-24,26)$ & Precisam de algum retreinamento & $53 \%(51 \%-55 \%)$ \\
\hline \multicolumn{4}{|c|}{ p-valor $<0,01$} \\
\hline \multicolumn{4}{|c|}{ PERCEPÇÃO RESULTADOS ACADÊMICOS - 32 pts } \\
\hline Ano & Média (IC 95\%) & Interpretação & Percentual $^{\mathrm{a}}(\mathrm{IC} 95 \%)$ \\
\hline $1^{\circ}$ ano & $17,76(16,38-19,14)$ & Sentindo-se mais pelo lado positivo & $56 \%(51 \%-60 \%)$ \\
\hline $3^{\circ}$ ano & $15,17(14,15-16,20)$ & Muitos aspectos negativos & $47 \%(44 \%-51 \%)$ \\
\hline $6^{\circ}$ ano & $15,04(13,26-16,82)$ & Muitos aspectos negativos & $47 \%(41 \%-53 \%)$ \\
\hline MÉDIA & $16,06(15,29-16,82)$ & Muitos aspectos negativos & $50 \%(48 \%-53 \%)$ \\
\hline \multicolumn{4}{|c|}{ p-valor $<0,01$} \\
\hline \multicolumn{4}{|c|}{ PERCEPÇÃO ATMOSFERA - 48 pts } \\
\hline Ano & Média (IC 95\%) & Interpretação & Percentual $^{\mathrm{a}}(\mathrm{IC} 95 \%)$ \\
\hline $1^{\circ}$ ano & $26,00(24,23-27,77)$ & Uma atitude mais positiva & $48 \%(44 \%-52 \%)$ \\
\hline $3^{\circ}$ ano & $22,07(20,43-23,72)$ & $\begin{array}{l}\text { Existem muitos problemas que precisam ser } \\
\text { mudados }\end{array}$ & $38 \%(35 \%-41 \%)$ \\
\hline $6^{\circ}$ ano & $21,65(18,10-25,21)$ & $\begin{array}{l}\text { Existem muitos problemas que precisam ser } \\
\text { mudados }\end{array}$ & $42 \%(36 \%-49 \%)$ \\
\hline MÉDIA & $23,38(22,20-24,56)$ & $\begin{array}{l}\text { Existem muitos problemas que precisam } \\
\text { ser mudados }\end{array}$ & $42 \%(40 \%-44 \%)$ \\
\hline \multicolumn{4}{|c|}{$\mathrm{p}$-valor $<0,001$} \\
\hline \multicolumn{4}{|c|}{ PERCEPÇÃO RELAÇÕES SOCIAIS - 28 pts } \\
\hline Ano & Média (IC 95\%) & Interpretação & Percentual $^{\mathrm{a}}(\mathrm{IC}$ 95\%) \\
\hline $1^{\circ}$ ano & $12,58(11,47-13,69)$ & Não é um lugar agradável & $45 \%(41 \%-49 \%)$ \\
\hline
\end{tabular}




\begin{tabular}{|clll|}
\hline $\mathbf{3}^{\mathbf{o}}$ ano & $12,60(11,73-13,47)$ & Não é um lugar agradável & $45 \%(42 \%-48 \%)$ \\
\hline $\mathbf{6}^{\mathbf{0}}$ ano & $13,00(11,32-14,68)$ & Não é um lugar agradável & $46 \%(40 \%-52 \%)$ \\
\hline MÉDIA & $12,66(12,04-13,28)$ & Não é um lugar agradável & $45 \%(43 \%-47 \%)$ \\
\hline & & p-valor $>0,05$ & \\
\hline
\end{tabular}

Nota. IC $=$ Intervalo de confiança

aPercentual de pontos do ano em relação ao total de pontos da dimensão

Fonte: Produzido pelos autores.

\subsection{Resultados de Itens Destacados}

Na pontuação individual dos itens, 32/50 afirmativas obtiveram média inferior a 2,0 pontos, indicando os pontos fracos na instituição e que necessitam de intervenção. Neste estudo, destacamos 22/50 itens do questionário relacionados a aspectos relevantes do ambiente educacional que consideramos associados ao desenvolvimento do ensino remoto (Quadro 2).

Quadro 2 - Média das pontuações de itens destacados e classificação

\begin{tabular}{|c|c|c|c|}
\hline $\mathrm{D}$ & Item & M & Classificação \\
\hline D1 & Sou estimulado(a) a participar das aulas & 1.5 & ponto fraco/ necessita intervenção \\
\hline D5 & Tenho estado muito cansado(a) para aproveitar este curso & 0.8 & ponto fraco / necessita intervenção \\
\hline D1 & O ensino adotado é frequentemente estimulante & 1.1 & ponto fraco / necessita intervenção \\
\hline D2 & Os professores ridicularizam os estudantes & 2.3 & aspecto que pode ser melhorado \\
\hline D2 & Os professores são autoritários & 1.9 & ponto fraco / necessita intervenção \\
\hline D1 & O ensino é centrado no estudante (mais auto aprendizado) & 1.9 & ponto fraco / necessita intervenção \\
\hline D5 & Tenho bons amigos(as) na Faculdade & 3,2 & ponto forte \\
\hline D4 & A prática de colar em provas é comum nesta Faculdade & 1.1 & ponto fraco / necessita intervenção \\
\hline D1 & O ensino é bastante coeso e focado & 1.5 & ponto fraco / necessita intervenção \\
\hline D1 & Sinto que venho sendo bem preparado(a) para a profissão & 1.9 & ponto fraco / necessita intervenção \\
\hline D1 & O método de ensino se preocupa em desenvolver minha confiança & 1.2 & ponto fraco / necessita intervenção \\
\hline D1 & O tempo para ensino é bem utilizado & 1.2 & ponto fraco / necessita intervenção \\
\hline D1 & O ensino enfatiza muito o aprendizado de fatos memorizáveis & 1.6 & ponto fraco / necessita intervenção \\
\hline D2 & Os professores dão um bom feedback aos estudantes & 1.4 & ponto fraco / necessita intervenção \\
\hline D2 & Os professores nos dão críticas construtivas & 1.9 & ponto fraco / necessita intervenção \\
\hline D4 & Tenho achado minha experiência aqui desapontadora & 2.1 & aspecto que pode ser melhorado \\
\hline D1 & Tenho certeza sobre os objetivos deste curso & 2.4 & aspecto que pode ser melhorado \\
\hline D4 & O ambiente me estimula a aprender & 1.6 & ponto fraco / necessita intervenção \\
\hline
\end{tabular}




\begin{tabular}{|c|c|c|c|} 
D1 & O ensino me encoraja a buscar meu próprio aprendizado & 2 & aspecto que pode ser melhorado \\
\hline D1 & A importância da educação continuada é enfatizada & 2.3 & aspecto que pode ser melhorado \\
\hline D1 & O ensino é muito centrado no professor & 1.5 & ponto fraco / necessita intervenção \\
\hline D4 & Me sinto à vontade para perguntar o que quero nas aulas & 1.9 & ponto fraco / necessita intervenção \\
\hline
\end{tabular}

Nota. D= Dimensão; M= média; D1= Dimensão Percepção do Ensino; D2=Dimensão Percepção dos Professores; D3=Dimensão Percepção dos Resultados acadêmicos; D4= Dimensão Atmosfera D5= Dimensão Relações sociais Fonte: Adaptado de Roff (2005).

\section{Discussão}

Os resultados evidenciam que à época da aplicação dos instrumentos - durante o ensino integralmente presencial, os estudantes obtiveram percepção negativa do curso de medicina. Alunos que não moravam com os pais, que antes de ingressar na graduação residiam fora do estado, e que levavam mais tempo para chegar à faculdade, tiveram percepção mais positiva do ambiente educacional, resultado que parece contradizer hipóteses que relacionam a percepção de suporte social mais robusto com satisfação com o curso de medicina (Enns et al., 2016). As variáveis gênero e faixa etária não foram estatisticamente significantes para a pontuação total, contrariando outros estudos (Enns et al., 2016; Genn \& Harden, 1986; McAleer \& Roff, 2001; Miles et al., 2012).

O ambiente educativo foi classificado com muitos problemas ("plenty of problems") apresentando diversos pontos fracos em todas as dimensões. A pontuação global decai ao longo da formação, indicando que ingressantes possuem percepção do curso mais positiva que alunos veteranos, o que corrobora estudos que verificaram decréscimo de entusiasmo, motivação e qualidade de vida do estudante ao longo da formação (Fernandes et al., 2019; Genn, 2001; Hutchinson, 2003). O ensino da escola em estudo é tradicional, o que pode explicar a baixa pontuação total: pesquisas que utilizaram o DREEM para comparar ambientes de aprendizagem com diferentes estratégias educacionais concluíram que alunos de instituições de metodologia inovadora têm percepções mais positivas do ambiente educativo do que estudantes de instituições de métodos de ensino tradicionais (Al-Hazimi et al., 2004; Zawawi \& Elzubeir, 2012).

Na dimensão "Ensino", os itens "o ensino adotado é frequentemente estimulante", "o ensino é bastante coeso e focado", "o método de ensino se preocupa em desenvolver minha confiança", "o tempo para ensino é bem utilizado" e "o ensino é muito centrado no professor" receberam as mais baixas pontuações, revelando que aspectos como motivação para estudar, uso adequado do tempo e incentivo à educação permanente foram pouco estimulados. Os alunos revelam que há pouca clareza quanto aos objetivos a serem alcançados, e a adoção de uma metodologia de ensino muito centrada no professor não favorece a participação ativa do aluno. Uma melhor definição sobre os objetivos do curso e estimular a busca do aprendizado são pontos que poderiam ser melhorados (Ministério da Educação do Brasil, 2014).

Na dimensão "Resultados Acadêmicos", os alunos revelam se sentir progressivamente inseguros quanto ao aprendizado adquirido. A percepção de capacidade de memorização decai e pode estar relacionada à dificuldade para reter grande quantidade de conteúdos cobrados em avaliações predominantemente somativas, características do modelo tradicional (Rodríguez et al., 2004).

Os resultados destacados revelaram uma insatisfatória interação professor-aluno em 2019. A dimensão "Professores", melhor avaliada no primeiro ano, foi em média apontada com necessidade de aprimoramento. Os itens com menores pontuações foram: “os professores dão um bom feedback aos estudantes” e "os professores são autoritários”. "Os professores nos dão críticas construtivas" e "os professores ridicularizam os estudantes" também obtiveram baixas pontuações. No ensino mais centrado no 
estudante, os métodos devem se basear nas experiências dos alunos (aprendizagem significativa) de forma que o ambiente seja colaborativo, com abertura ao diálogo e livre de críticas ou humilhações, e os estudantes possam sentir-se confiantes para expressar dúvidas, identificar lacunas de conhecimento, testar limites (Hutchinson, 2003), e, em uma concepção freiriana (Freire, 2005), desenvolver espírito crítico e transformador.

A atmosfera do curso foi percebida pelos alunos com "muitos problemas que precisam ser mudados". O ambiente não os estimula a aprender e eles não se sentem à vontade para fazer perguntas nas aulas, percebendo a experiência da formação como "desapontadora". Tal resultado pode ser explicado pelo fato de o modelo tradicional de ensino exigir que os alunos frequentem aulas monótonas, longas e cansativas, com poucas oportunidades para participarem e se envolverem em discussões. Isto pode criar uma sensação de tédio durante as aulas e fazer com que os alunos se sintam menos motivados (Alsoufi et al., 2020). O desânimo e a sensação de exaustão do aluno, além de representarem fatores de risco para a emergência de transtornos psíquicos (Dyrbye et al., 2021), também podem levar ao abandono do curso e ao absenteísmo, preocupações para muitas universidades em todo o mundo (Alsoufi et al., 2020; Emanuel, 2020). Cabe ressaltar que o item "Tenho estado muito cansado(a) para aproveitar este curso" obteve a pontuação média mais baixa do questionário, revelando que essa sensação foi verificada em todos os anos estudados. O sentimento crescente de desgaste e exaustão emocional sofridas pelo estudante de medicina corrobora os estudos voltados à saúde mental do discente (Dyrbye et al., 2021; Rotenstein et al., 2016; ). Ainda que evidências se refiram à prevalência de ansiedade, depressão e síndrome de burnout (Damiano, de Oliveira, Ezequiel, AL Lucchetti \& G Lucchetti, 2021; Dyrbye, Satele \& West, 2021) em ambientes presenciais, também permanecem como objeto de preocupação em ambientes virtuais (Maia \& Dias, 2020), e sua ocorrência neste formato de ensino não pode ser negligenciada.

Em relação à dimensão Relações Sociais, apesar da faculdade ter sido percebida como um "lugar não agradável" em momento anterior à crise sanitária, os alunos avaliaram como ponto mais forte do ambiente a possibilidade de construção de laços de amizade ("Tenho bons amigos na faculdade"), de forma que há um campo profícuo e receptivo para iniciativas que estimulem os vínculos pessoais. Para o desenvolvimento de atividades remotas, além de recursos de comunicação que garantam a interatividade, as relações sociais e o sentimento de fazer parte de um grupo precisam ser fomentados como forma de evitar o isolamento e proporcionar a cooperação entre os estudantes (Ministério da Educação do Brasil, 2007). São promissoras as atividades que incentivem a criação de vínculos entre alunos e professores e fortaleçam o sentimento de pertencimento institucional, mesmo que realizadas de forma online (Serra et al., 2021).

\subsection{O que é preciso mudar?}

Os resultados da pesquisa apontaram que, apesar das mudanças preconizadas pelas DCNs, diversos aspectos do ambiente educacional necessitam de intervenção, pois permanecem estruturados no modelo tradicional de ensino. Parece fundamental a revisão destes aspectos e a superação deste modelo para que a educação médica esteja em conformidade com as demandas atuais da formação, principalmente pela necessidade de incorporação de tecnologias de informação e comunicação (TIC).

Em 1998, a UNESCO já observava que o uso mais ampliado da tecnologia causaria impacto na educação, afirmando a potencialidade observada nas TIC para renovar o conteúdo dos cursos e o modo como o conhecimento é desenvolvido, adquirido e transmitido. Cabe ressaltar que a potencialidade para transformar a educação não reside apenas no uso instrumental das TICs, mas nas propostas pedagógicas a partir das quais se viabiliza a sua utilização, como o desenvolvimento de competências de comunicação e de pensamento crítico como fundamentos para a busca seletiva de informações fidedignas nos ambientes digitais. Em ambientes virtuais de aprendizagem (AVA), é fundamental que os participantes interajam (Alves, 2020), sendo coautores do processo de aprendizagem, através do exercício dialógico que transforma a informação em conhecimento (Organização das 
Nações Unidas para Educação, Ciência e Cultura [UNESCO], 1998). Entretanto, o que se tem observado na vigência do ensino remoto é a adaptação de metodologias utilizadas no modo presencial adaptadas ao ambiente virtual, com aulas realizadas nos horários pré-estabelecidos e apresentação de aulas expositivas com os mesmos professores responsáveis pelas disciplinas (Alves, 2020). Observa-se um uso instrumental das tecnologias nos padrões e princípios do modelo tradicional, onde continua a se praticar uma "educação bancária" (Freire, 2005) na qual os docentes "depositam" os conteúdos sob alunos passivos, reproduzindo relações verticalizadas e pouco instigantes à reflexões e questionamentos.

A adequação do ensino à modalidade remota exige repensar os lugares ocupados por professores e alunos, principalmente nas escolas de metodologia tradicional (Coll \& Monereo, 2010). Relações pautadas na hierarquia e no autoritarismo dificultam parcerias necessárias ao trabalho colaborativo e não se alinham aos objetivos de um projeto pedagógico baseado na construção partilhada do conhecimento, base conceitual de métodos mais inovadores (Freire, 2005; Venturelli, 1997).

A inevitável incorporação do ensino remoto e o uso das TICs na educação médica têm a potencialidade para revigorar os preceitos contidos nas DCNs e impulsionar mudanças curriculares, reorganizando processos de ensino-aprendizagem (Goudouris \& Struchiner, 2015) que contemplem o ensino em diversos cenários. A pandemia e seus efeitos na educação médica reafirmaram a necessidade de se formar profissionais críticos e ao mesmo tempo criativos, com capacidade de tomar o conhecimento como algo a ser construído e desconstruído continuamente, em sintonia com a realidade e com as demandas que dela advém. Apesar do consenso sobre a importância da avaliação institucional, as investigações sobre as perspectivas dos alunos ocupam pouco espaço na literatura e esta pesquisa permitiu valorizá-las ao participarem dos processos de mudança. Estabelecer um diálogo entre os dados recolhidos, gestão institucional e corpo docente, e fomentar reflexão e diálogo em busca de mudanças pactuadas representa um instigante desafio.

\section{Conclusão}

Esta pesquisa revelou ser fundamental acompanhar o processo de rupturas e mudanças das práticas educativas e principalmente as transformações do ambiente educacional na nossa instituição. Os resultados deste estudo, apesar de limitados à percepção do curso médico por parte de um de seus componentes - os alunos, configuram uma valiosa fonte de dados que permitirão estudos comparativos que acompanhem as inovações introduzidas, as dinâmicas utilizadas e as implicações pedagógicas frente às demandas de renovação, ou mesmo estudos de natureza qualitativa, que investiguem de forma mais aprofundada as percepções de estudantes de medicina diante das transformações em curso. Novas investigações se fazem necessárias para complementar a compreensão do atual momento da formação médica. Em uma perspectiva futura, pretendemos desenvolver um novo estudo pautado no objetivo de conhecer as percepções dos alunos e professores após as transformações efetivadas pelo ensino remoto emergencial. Buscaremos realizar um estudo comparativo entre as percepções sobre a formação acadêmica no momento prévio à eclosão da crise sanitária e aquelas a serem reveladas no estudo proposto, estabelecendo um diálogo entre elas. Aspectos como a diversificação de estratégias metodológicas que coloquem o aluno na centralidade do processo de ensino-aprendizagem, a readequação de processos avaliativos, a definição sobre os conteúdos essenciais à formação médica, e o investimento no desenvolvimento docente, nos pareceram pontos-chave a serem aprofundados e tomados como critérios comparativos na nova investigação.

Por fim, a necessidade de mudanças na educação médica foi renovada pelo uso de plataformas digitais, aspecto não contemplado no questionário DREEM. Diante dos novos cenários de ensino-aprendizagem, o desenvolvimento de instrumentos e métodos mais adequados à avaliação das percepções do ensino mediado por tecnologias, nos daria informações mais precisas para subsidiar o planejamento de intervenções. 


\section{Referências}

Al-Hazimi, A., Zaini, R., Al-Hyiani, A., Hassan, N., Gunaid, A., Ponnamperuma, G., Karunathilake, I., Roff, S., McAleer, S., \& Davis. (2004). Educational environment in traditional and innovative medical schools: a study in four undergraduate medical schools. Educ Health (Abingdon); 17 (2), 192-203. https://doi.org/10.1080/13576280410001711003

Alsoufi, A., Alsuyihili, A., Msherghi, A., Elhadi, A., Atiyah, H., Ashini, A., Ashwieb, A., Ghula, M., Ben Hasan, H., Abudabuos, S., Alameen, H., Abokhdhir, T., Anaiba, M., Nagib, T., Shuwayyah, A., Benothman, R., Arrefae, G., Alkhwayildi, A., Alhadi, A., Zaid, A., \& Elhadi, M. (2020). Impact of the COVID-19 pandemic on medical education: Medical students' knowledge, attitudes, and practices regarding electronic learning. PloS one, 15(11), e0242905. https://doi.org/10.1371/journal.pone.0242905

Alves, L. (2020). Educação remota: entre a ilusão e a realidade. EDU, 8 (3), 348-65. https://periodicos.set.edu.br/educacao/article/view/9251

Behrens, M. A.(1999). A prática pedagógica e o desafio do paradigma emergente. R. bras. Est. pedag., 80 (196), $383-403$.

Coll, C., \& Monereo, C. Educação e Aprendizagem no século XXI: novas ferramentas, novos cenários, novas finalidades. (2010). In: Coll C, Monereo C (org.). Psicologia da educação virtual: aprender e ensinar com as tecnologias da informação e da comunicação. (15-46). Artmed.

Damiano, R. F., de Oliveira, I. N., Ezequiel, O. S., Lucchetti, A. L., \& Lucchetti, G. (2021). The root of the problem: identifying major sources of stress in Brazilian medical students and developing the Medical Student Stress Factor Scale. Braz. J. Psychiatr. 43 (1), 35-42. https://doi.org/10.1590/1516-4446-20190824

Dost, S., Hossain, A., Shehab, M., Abdelwahed, A., \& Al-Nusair, L. (2020). Perceptions of medical students towards online teaching during the COVID-19 pandemic: a national cross-sectional survey of $2721 \mathrm{UK}$ medical students. BMJ open, 10(11), e042378. https://doi.org/10.1136/bmjopen-2020-042378

Dyrbye, L. N., Satele, D., \& West, C. P. (2021). Association of Characteristics of the Learning Environment and US Medical Student Burnout, Empathy, and Career Regret. JAMA network open, 4(8), e2119110. https://doi.org/10.1001/jamanetworkopen.2021.19110

Emanuel E. J. (2020). The Inevitable Reimagining of Medical Education. JAMA, 323(12), 1127-1128. https://doi.org/10.1001/jama.2020.1227

Enns, S. C., Perotta, B., Paro, H. B., Gannam, S., Peleias, M., Mayer, F. B., Santos, I. S., Menezes, M., Senger, M. H., Barelli, C., Silveira, P. S., Martins, M. A., \& Zen Tempski, P. (2016). Medical Students' Perception of Their Educational Environment and Quality of Life: Is There a Positive Association? Academic medicine: journal of the Association of American Medical Colleges, 91(3), 409-417. https://doi.org/10.1097/ACM.0000000000000952

Fernandes, D. A. S., Taquette, S. R., Rodrigues, N. C. P. (2019). The educational environment of a traditional public school of medicine in Brazil with the DREEM questionnaire. MedEdPublish, 8, 31-56. https://doi.org/10.15694/mep.2019.000101.1

Freire, P. (2005). Pedagogia do oprimido. (42a ed.), Paz e Terra.

Frenk, J., Chen, L., Bhutta, Z. A., Cohen, J., Crisp, N., Evans, T., Fineberg, H., Garcia, P., Ke, Y., Kelley, P., Kistnasamy, B., Meleis, A., Naylor, D., PablosMendez, A., Reddy, S., Scrimshaw, S., Sepulveda, J., Serwadda, D., \& Zurayk, H. (2010). Health professionals for a new century: transforming education to strengthen health systems in an interdependent world. Lancet (London, England), 376(9756), 1923-1958. https://doi.org/10.1016/S0140-6736(10)61854-5

Genn J. M. (2001). AMEE Medical Education Guide No. 23 (Part 1): Curriculum, environment, climate, quality and change in medical education-a unifying perspective. Medical teacher, 23(4), 337-344. https://doi.org/10.1080/01421590120063330.

Genn, J. M., \& Harden, R. M. (1986). What is medical education here really like? Suggestions for action research studies of climates of medical education environments. Medical teacher, 8(2), 111-124. https://doi.org/10.3109/01421598609010737

Gil, A. C. (2016). Métodos e Técnicas de Pesquisa Social, (6a ed.), Atlas.

Gordon, M., Patricio, M., Horne, L., Muston, A., Alston, S. R., Pammi, M., Thammasitboon, S., Park, S., Pawlikowska, T., Rees, E. L., Doyle, A. J., \& Daniel, M. (2020). Developments in medical education in response to the COVID-19 pandemic: A rapid BEME systematic review: BEME Guide No. 63. Medical teacher, 42(11), 1202-1215. https://doi.org/10.1080/0142159X.2020.1807484

Goudouris, E., \& Struchiner, M. (2015). Aprendizagem Híbrida na Educação Médica: uma Revisão Sistemática. Rev. bras. educ. med., 39 (4), 620-629. https://doi.org/10.1590/1981-52712015v39n4e01642014.

Hutchinson L. (2003). Educational environment. BMJ (Clinical research ed.), 326(7393), 810-812. https://doi.org/10.1136/bmj.326.7393.810

Magalhães, A. J. A, Rocha, M. H. A, Santos, S. C., Dantas, C. B., Manso, G. J. M. C., \& Ferreira, M. D. A. (2020). O Ensino da Anamnese Assistido por Tecnologias Digitais durante a Pandemia da Covid-19 no Brasil. Rev. bras. educ. med, 44 (Suppl 01), https://doi.org/10.1590/1981-5271v44.supl.1-20200437.

Maia, B. R, \& Dias, P. C. (2020). Ansiedade, depressão e estresse em estudantes universitários: o impacto da COVID-19. Estud. psicol., 37, e200067. https://doi.org/10.1590/1982-0275202037e200067.

McAleer, S., \& Roff, S. (2001). A practical guide to using the Dundee Ready Education Environment Measure (DREEM). In J. M. Genn (Ed.), Curriculum, environment, climate, quality and change in medical education: A unifying perspective (pp. 29-33). AMEE Education Guide no. 23. Scotland: AMEE.

Miles, S., Swift, L., \& Leinster, S. J. (2012). The Dundee Ready Education Environment Measure (DREEM): a review of its adoption and use. Medical teacher, 34(9), e620-e634. https://doi.org/10.3109/0142159X.2012.668625 
Ministério da Educação do Brasil. (2007). Referenciais de qualidade para Educação Superior à Distância. http://portal.mec.gov.br/auditorias/193-secretarias112877938/seed-educacao-a-distancia-96734370/12777-referenciais-de-qualidade-para-ead.

Ministério da Educação do Brasil. (2014). Resolução n 3 3, de 20 de Junho de 2014. Diretrizes Curriculares Nacionais do Curso de Graduação em Medicina. http://portal.mec.gov.br/index.php?option=com_docman\&view=download\&alias=15874-rces003-14\&category_slug=junho-2014-pdf\&Itemid=30192

O'Doherty, D., Dromey, M., Lougheed, J., Hannigan, A., Last, J., \& McGrath, D. (2018). Barriers and solutions to online learning in medical education - an integrative review. BMC medical education, 18(1), 130. https://doi.org/10.1186/s12909-018-1240-0

Organização das Nações Unidas para Educação, Ciência e Cultura [UNESCO]. (1998). Declaração Mundial sobre Educação Superior no Século XXI: Visão e Ação. Comunicado. Paris, UNESCO.

Organização das Nações Unidas para Educação, Ciência e Cultura [UNESCO]. (2008). Padrões de competência em TIC para professores: módulos de padrão de competências. Paris: UNESCO.

Organização das Nações Unidas para Educação, Ciência e Cultura [UNESCO]. (2013). Enfoques estratégicos sobre las TICs en educación en América Latina y el Caribe: Medición de aprendizaje y nuevas prácticas educativas. Santiago: Oficina Regional de Educación para América Latina y el Caribe. http://www.unesco.org/new/fileadmin/MULTIMEDIA/FIELD/Santiago/images/ticsesp.pdf

Rodrigues, B. B, Cardoso, R. R. J., Peres, C. H. R., \& Marques, F. F. (2020). Aprendendo com o imprevisível: saúde mental dos universitários e educação médica na pandemia de covid-19. Rev Bras Educ Med, 44(1), 1-5. https://doi.org/10.1590/1981-5271v44.supl.1-20200404

Rodríguez, C. A., Poli, P, \& Behrens, M. A. (2004). Paradigmas Educacionais e a Formação Médica. Revista Brasileira de Educação Médica, 28 (03), 234-241. https://doi.org/10.1590/1981-5271v28.3-030>.

Roff, S., \& McAleer, S. (2001). What is educational climate? Medical teacher, 23(4), pp. 333-334. https://doi.org/10.1080/01421590120063312

Roff S. (2005). The Dundee Ready Educational Environment Measure (DREEM)--a generic instrument for measuring students' perceptions of undergraduate health professions curricula. Medical teacher, 27(4), pp. 322-325. https://doi.org/10.1080/01421590500151054

Roff, S.,McAleer, S., Harden, R. M., Al-Qahtani,M., Ahmed, R. U., Deza, H., Groenen, G. \& Primparyon, P. (1997) Development and validation of the Dundee Ready Education Environment Measure (DREEM), Medical Teacher, 19:4, 295-299. https://doi.org/10.3109/01421599709034208

Rotenstein, L. S., Ramos, M. A., Torre, M., Segal, J. B., Peluso, M. J., Guille, C., Sen, S., \& Mata, D. A. (2016). Prevalence of Depression, Depressive Symptoms, and Suicidal Ideation Among Medical Students: A Systematic Review and Meta-Analysis. JAMA, 316(21), 2214-2236. https://doi.org/10.1001/jama.2016.17324

Ruiz, J. G., Mintzer, M. J., \& Leipzig, R. M. (2006). The impact of E-learning in medical education. Academic medicine : journal of the Association of American Medical Colleges, 81(3), 207-212. https://doi.org/10.1097/00001888-200603000-00002

Serra, S. T., Bteshe, M., Bedirian, R., Belz, D. S., Franco, C. F., \& de Oliveira, L. S. S. (2021). Implantação de mentoria on-line em uma faculdade de medicina durante a pandemia da Covid-19. Rev. bras. educ. med. 45 (suppl 1), e127. https://doi.org/10.1590/1981-5271v45.supl.1-20210193.

Taquette, S. R., Borges, L. (2020). Pesquisa qualitativa para todos. Vozes.

Troncon, L. E. de A. (2014). Ambiente educacional. Medicina (Ribeirão Preto), 47(3), 264-271. https://doi.org/10.11606/issn.2176-7262.v47i3p264-271

Venturelli, J. (1997). Educación médica: nuevos enfoques, metas y métodos [Internet]. Washington: OPAS. https://www.imperial.ac.uk/media/imperialcollege/administration-and-support-services/library/public/vancouver.pdf

Zawawi, A. H., \& Elzubeir, M. (2012). Using DREEM to compare graduating students' perceptions of learning environments at medical schools adopting contrasting educational strategies. Medical teacher, 34 Suppl 1, S25-S31. https://doi.org/10.3109/0142159X.2012.656747 